Natural Hazards and Environmental Decision Making

\title{
Risk Assessment of Cut Slope Failure Due to Heavy Rainfall by Machine Learning; Case Study on Expressway in Japan Based on Actual Damage Experiences
}

\author{
Nagao Kazuyuki ${ }^{1}$, Sawano Koki ${ }^{2}$, Haga Natsumi ${ }^{3}$, Kamura Akiyoshi $^{3}$, and Kazama Motoki ${ }^{3}$ \\ ${ }^{1}$ East Nippon Expressway Co., Ltd., 100-8979, Tokyo, Japan \\ ${ }^{2}$ Nexco Engineering Tohoku, 980-0013, Sendai, Japan \\ ${ }^{3}$ Tohoku University, 980-8579, Sendai, Japan
}

doi: https://doi.org/10.21467/abstracts.93.40

\section{ABSTRACT}

There are many cut and fill slopes in expressway in Japan, because those are located at the mountain areas. Recently, due to heavy rainfall or large-scale earthquakes, the collapses of slopes on expressways have been seriously influenced to economic activities and daily lives (Nagao et al., 2019, Haga et al., 2019). Furthermore, about $40 \%$ of expressways network are over 30 years in service, and the deterioration risk becomes also higher than ever before. In this paper, we introduce the risk evaluation strategy of the cut slopes due to heavy rainfall based on actual damage data in Japan.

Damage of the slopes due to heavy rainfall is affected by both rainfall pattern and the characteristics of slopes such as ground material, slope angle, slope size, operation period and topography. Firstly, we studied the rainfall pattern at the collapse point of the cut slope based on actual experiences from the analytical rainfallprovided by the Japan Meteorological Agency, which is the "trigger". Secondary, we scored many factors, such as topography, geology based on the slope data base. When we gave evaluation values to each slope, the frequency distribution regarding the evaluation value was obtained as shown in the Figures 1 and 2. Number of damage data 92 is very small comparing to the that of non-damage data 6812 for Tohoku district in Japan during the 1993 to 2017 . Figures indicate that the larger the numerical value, the greater the risk. Details of how to calculate the evaluation value are omitted here. It suffices if the evaluation value serving as the threshold value is properly determined based on the actual damage data, but generally it is not as shown in Figures 1 and 2. The reason why is that it is very difficult to extract the unique index even using multivariate analysis, because there are a lot of factors that contribute to slope failure. This is because it is difficult to determine which index should be weighted and how much.In such a case, machine learning is very effective to assess weight of each risk index.

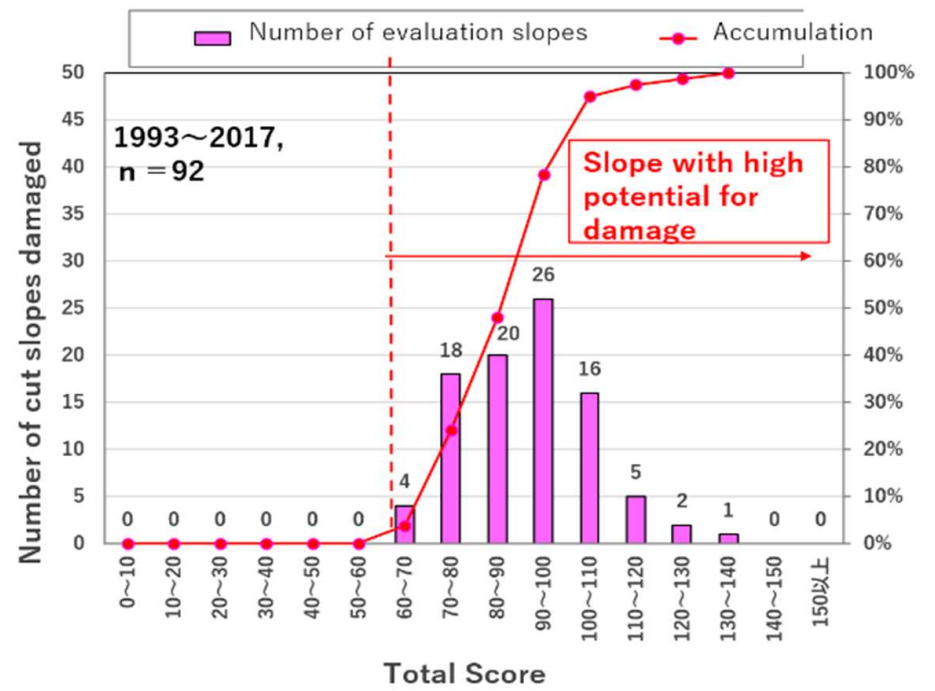

Fig.1 Frequency distribution and the cumulative curve of cut slopes actually damaged by heavy rainfall, in Tohoku district in Japan, during 1993 to 2017 period. 
The Second Eurasian RISK-2020 Conference and Symposium

Figure 3 shows the schematic diagram of the comparison between the risk assessment method and the machine learning using artificial intelligence (AI). In the right hand side of the figure, actual evaluation results is shown. In this case, we obtained good results for predicting the damage risk of cut slopes due to heavy rainfall based on the actual damage data. By using the AI function, we can evaluate the damage risk due to heavy rainfall in real time and which slope is vulnerable beforehand.

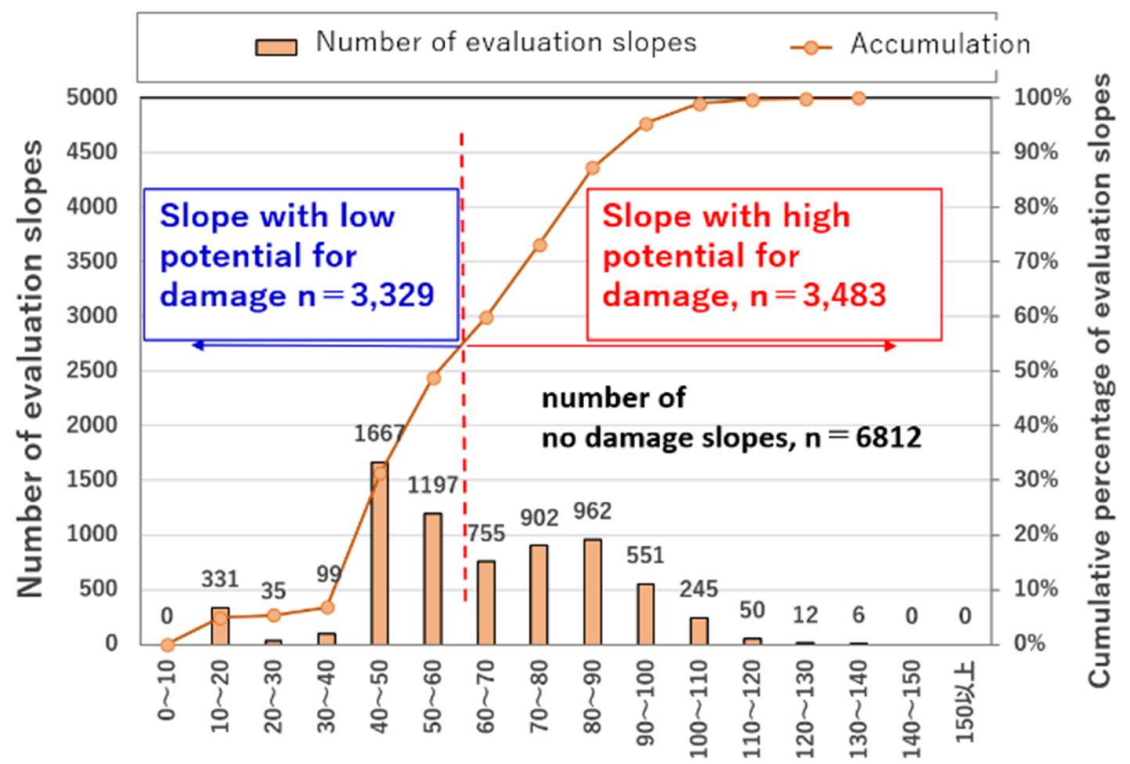

Total Score

Fig.2 Frequency distribution and cumulative curve of cut slope non-damaged by heavy rainfall, in Tohoku district in Japan, during 1993 to 2017 period

a) Conventional method using multivariate analysis.

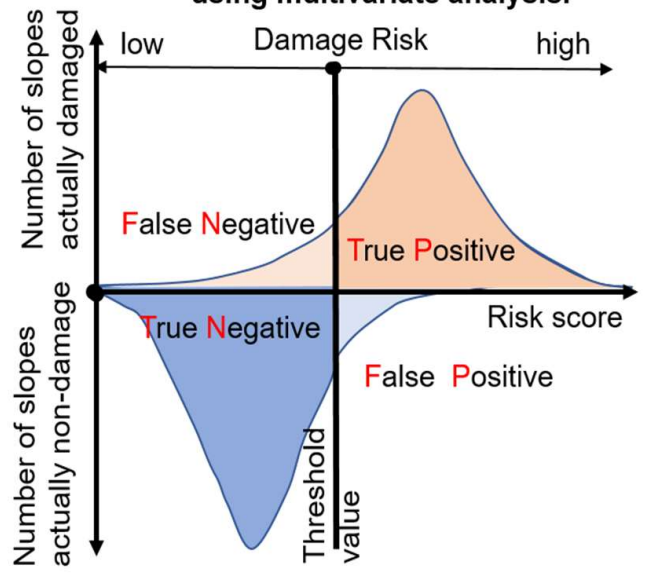

b) Risk assessment using Machine learning

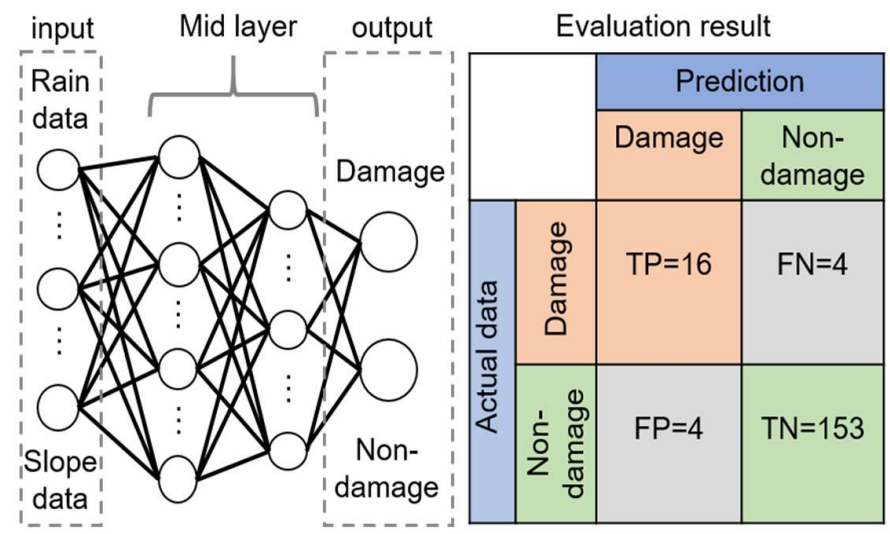

Fig.3 Schematic diagram of the confusion matrix of the risk evaluation for highway cut slopes.

\section{References}

1) Nagao, K., Sawano, K., Matsuzaki, K., Kazama, M., Kawai, T., and Kamura, A.: Case study of highway slope damage caused by heavy rain and its characteristics of Tohoku district. Journal of JSCE, Division C: Geotechnics, Vol.75, No.2, pp.198-215, 2019.

2) Haga, N. , Kazama, M., Kamura, A., Nagao, K. , Usami, M. and Sawano, K. : Cut slope damage of expressway caused by heavy rainfall in Tohoku district, Japan, pp.ATC3-4, pdf, 4p. 2019. 